http://dx.doi.org/10.1590/1678-4162-8859

Arq. Bras. Med. Vet. Zootec., v.68, n.6, p.1573-1580, 2016

\title{
Efeito da suplementação com colina protegida sobre parâmetros bioquímicos, produção e reprodução de vacas leiteiras no periparto
}

[Effect of protected choline supplementation on biochemical parameters, production, and reproduction of dairy cows in peripartum]

\author{
A.R. Aires ${ }^{1}$, X.R. Rocha ${ }^{2}$, V.D. Torbitz ${ }^{1}$, R. Moresco ${ }^{1}$, R.S. Sousa ${ }^{3}$, S.L.S. Severo ${ }^{2}$, W. Naibo ${ }^{2}$, \\ R.A. Sossanovicz ${ }^{2}$, A. Pretto ${ }^{1}$, E.L. Ortolani ${ }^{4}$, M.L.R. Leal ${ }^{1}$ \\ ${ }^{1}$ Universidade Federal de Santa Maria - UFSM - Santa Maria, RS \\ ${ }^{2}$ Universidade do Oeste de Santa Catarina - Unoesc - Xanxerê, SC \\ ${ }^{3}$ Universidade de São Paulo - USP - São Paulo, SP \\ ${ }^{4}$ Instituto Federal Catarinense - IFC - Concórdia, SC
}

\begin{abstract}
RESUMO
Avaliou-se o efeito da suplementação com colina protegida sobre o perfil energético, as enzimas hepáticas e a reprodução de vacas leiteiras no periparto. Quinze vacas leiteiras foram divididas em dois grupos experimentais: oito receberam 80 gramas de colina protegida por 21 dias no pré-parto e por 40 dias no pós-parto e sete foram consideradas controle. Amostras de sangue foram coletadas nos dias 10, 20, 30 e 60 pós-parto para avaliação dos perfis energético e hepático. Aos 60 dias pós-parto, realizou-se exame ginecológico dos animais para avaliação da saúde reprodutiva. A suplementação com colina protegida não alterou os níveis de beta-hidroxibutirato (BHBA), ácidos graxos não esterificados (AGNE), frutosamina, fator de crescimento semelhante a glicose I (IGF-I), status oxidante total (TOS), aspartato aminotransferase (AST) e gamaglutamiltransferase (GGT) no pós-parto. Não houve diferença também quanto à produção de leite. Aos 60 dias pós-parto, vacas suplementadas com colina protegida apresentaram menor número de casos de endometrite que vacas do grupo controle. A suplementação de colina protegida não alterou o perfil bioquímico e a produção de leite, mas reduziu o número de casos de endometrite no pós-parto de vacas leiteiras.
\end{abstract}

Palavras-chave: período de transição, vacas leiteiras, parâmetros bioquímicos, colina protegida

\begin{abstract}
The study aimed to evaluate the effect of supplementation with protected choline on the energy profile, liver enzymes and reproduction in dairy cows in peripartum. Fifteen cows were divided into two groups: 8 received 80 grams of protected choline for 21 days pre-partum and 40 days postpartum, and 7 were considered control. Blood samples were collected on days 10, 20, 30, and 60 postpartum to evaluate the energy and hepatic profiles. After 60 days postpartum a gynecological examination of animals for evaluation of reproductive health was done. The supplementation with choline protected did not alter the beta-hydroxybutyrate levels (BHBA), non-esterified fatty acids (NEFA), fructosamine, like growth factor glucose (IGF-I), total oxidant status (TOS), aspartate aminotransferase (AST) and glutamiltrasferase range (GGT) postpartum. There was no difference in milk production. After 60 days postpartum, cows fed protected choline had fewer cases of endometritis that cows in the control group. The protected choline supplementation did not alter the biochemical profile and milk production, but reduced the number of endometritis cases in postpartum dairy cows.
\end{abstract}

Keywords: Transition period, dairy cows, biochemical parameters, rumen protected choline

Recebido em 6 de novembro de 2015

Aceito em 20 de abril de 2016

E-mail: adelina_ravet@yahoo.com.br 


\section{INTRODUÇÃO}

Com o aumento na produção de leite observado nas últimas décadas, as alterações metabólicas, experimentadas pela vaca leiteira no periparto, tornaram-se cada vez mais evidentes, fazendo do período de transição o principal desafio para o animal e para o produtor. Durante o período de transição, que corresponde aos 21 dias antes do parto e aos 21 dias de lactação, a ingestão de matéria seca diminui drasticamente, enquanto as exigências nutricionais da vaca leiteira praticamente dobram (Nutrient..., 2001), principalmente no início da lactação. Como resultado dessa equação, as vacas nesse período, invariavelmente, encontram-se em balanço energético negativo (BEN).

$\mathrm{O}$ balanço energético negativo tem como principal consequência metabólica a mobilização das reservas corporais de energia na forma de ácidos graxos não esterificados (AGNEs), sendo este o substrato hepático para produção de energia para praticamente todas as reações do organismo em desbalanço (Drackley et al., 2001). Sabe-se que quanto mais AGNEs são mobilizados das reservas, maior é a produção de seus metabólitos no fígado e maior são as chances de ocorrência de transtornos no periparto (Duffield et al., 2009).

Um período de transição realizado com sucesso é aquele em que a vaca consegue compensar o BEN sem graves alterações produtivas e de saúde. A colina é uma quasi-vitamina, que apresenta funções importantes no organismo dos animais. Proveniente da metionina, a colina é requerida para síntese de fosfatidilcolina, fosfolipídio abundante em mamíferos, fundamental para ações como absorção e transporte de lipídios, fazendo parte da estrutura da membrana celular, da sinalização celular e da síntese de lipoproteínas (Zeisel e HolmesMcNary, 2001).

A deficiência de colina está relacionada com a ocorrência de lipidose hepática (Zeisel e HolmesMcNary, 2001), uma vez que os triglicerídeos são transportados para fora do fígado como um constituinte de lipoproteína de baixo peso molecular (VLDL), extremamente dependente de colina (Piepenbrink e Overton, 2003). A suplementação de colina durante o periparto de vacas leiteiras tem sido realizada em intevalos e doses bem variáveis, apresentado efeitos controversos sobre o desempenho produtivo, a saúde e a reprodução pós-parto (Xu et al., 2006; Cooke et al., 2007; Lima et al., 2012). O objetivo do presente estudo foi avaliar o efeito da suplementação com colina protegida sobre o desempenho produtivo, o perfil metabólico, as enzimas hepáticas e a ocorrência de endometrite de vacas no periparto.

\section{MATERIAL E MÉTODOS}

Foram selecionadas 15 vacas leiteiras da raça Holandesa, entre a segunda e a quarta lactação, criadas em sistema semi-intensivo, alocadas em uma propriedade de médio porte localizada no município de Xanxerê, região Oeste de Santa Catarina. Foram selecionadas para participar do experimento apenas vacas com escore corporal entre 3 e 4 . As vacas passaram por exame clínico completo antes do início e durante o experimento, e as que apresentaram alteração clínica foram excluídas. A dieta durante o período pré-parto foi composta por silagem de milho, ração comercial e sal aniônico à base de cloreto de amônia ( $1 \%$ da ração); nessa fase, as vacas foram mantidas em área de pouca oferta de pastagem de tifton. No período pós-parto, a alimentação foi à base de silagem de milho $(7 \mathrm{~kg}$ de matéria seca por dia), pastagem de milheto (à vontade), ração comercial (22\% de proteína) ( $9 \mathrm{~kg}$ de $\mathrm{ração} / \mathrm{vaca} / \mathrm{dia})$.

Os animais selecionados foram divididos em dois grupos: controle, composto por sete vacas, e tratado, composto por oito vacas. Os animais pertencentes ao grupo tratado receberam diariamente $80 \mathrm{~g}$ de colina protegida (Toplac Transição - Nutrifarma, Taió, Brasil). O período de tratamento iniciou 21 dias antes do parto até 40 dias após o parto. Durante o pré-parto, o tratamento foi fornecido pela manhã, individualmente, junto à ração e ao sal aniônico. A colina protegida foi fornecida após a ordenha da manhã, individualmente, adicionada à ração.

No período pré-parto, as vacas foram monitoradas quanto à efetividade da dieta aniônica, mediante mensuração do $\mathrm{pH}$ urinário. A urina foi coletada nos dias 21 e 10, anteriores à data prevista para o parto, por meio de estímulo manual na região do períneo, e o $\mathrm{pH}$ urinário foi mensurado utilizando-se pHmetro digital portátil (PH1800 - Instrutherm, São Paulo, SP). Para descartar a influência da hipocalcemia sobre o desempenho pós-parto, animais com $\mathrm{pH}$ superior 
a 7 nas duas mensurações foram retirados do experimento.

Nos dias 21 e 10, do período pré-parto, foram realizadas coletas de sangue, obtidas por punção da veia coccígea, para determinação dos teores de beta-hidroxibutirato (BHBA). Concentrações desse metabólito superiores a $3 \mathrm{mmol} / \mathrm{L}$ no pré-parto indicam cetose clínica (Oetzel, 2004), e animais nessa condição foram excluídos do experimento.

Amostras de sangue foram coletadas da veia coccígea nos dias 10,20, 30 e 60 pós-parto. Após a coleta, o sangue foi centrifugado por 10 minutos a 6000 rotações/minuto para obtenção do soro, que foi congelado a $-20^{\circ} \mathrm{C}$ até o momento das análises.

Foram determinados, por meio de métodos colorimétricos, os teores séricos de frutosamina, de colesterol e das enzimas aspartato aminotransferase (AST) e gamaglutamiltransferase (GGT), utilizando-se kit comercial (Bioclin), assim como as concentrações séricas dos ácidos graxos não esterificados (kit comercial Wako), e do betahidroxibutirato (kit comercial Randox). O status oxidativo total foi determinado de acordo com a técnica descrita por Erel (2004). As análises foram realizadas em analisador automático bioquímico (Cobas Mira - Roche Diagnostics, Montclair, EUA). As concentrações do fator de crescimento semelhante à insulina tipo 1 (IGF-1) foram determinadas por eletroquimioluminescência em equipamento Immulite 1 (Siemens, modelo 1000, São Paulo, SP, Brasil). Nas amostras coletadas aos 60 dias pós-parto, determinaram-se os teores de progesterona, por radioimunoensaio e de betahidroxibutirato e IGF-1, como descrito anteriormente.

A produção de leite individual foi mensurada nos dias 10,20 e 30 do experimento, utilizando-se coletor de leite acoplado ao sistema de ordenha. $\mathrm{O}$ exame ginecológico no pós-parto foi realizado aos 60 dias de lactação, mediante técnica descrita por Grunert e Gregory (1989), por meio de palpação retal e vaginoscopia. Informações relativas à data da primeira inseminação e à data de concepção pósparto foram obtidas nas fichas de acompanhamento individual para avaliação do desempenho reprodutivo.

O protocolo experimental foi aprovado pela Comissão de Ética em Uso de Animais da
Universidade Federal de Santa Maria (108/2013). Os dados foram testados quanto à normalidade utilizando-se o teste de Kolmogorov-Smirnov. Parâmetros com distribuição não paramétrica passaram por transformação logarítmica $(\log (10)+1)$. O efeito do tratamento, do tempo e da interação desses dois fatores sobre os parâmetros sanguíneos foi analisado pelo comando proc mixed (Statistical..., 2000). As médias ajustadas (LSMeans) dos fatores fixos foram comparadas pela diferença mínima significativa (LSD), obtida pelo teste " $\mathrm{t}$ " a um nível de significância de $5 \%$. Para o intervalo entre parto e concepção, foi realizada comparação de médias por meio do teste de Tukey com nível de significância de 5\%. Os resultados foram expressos em médias \pm erropadrão. Os dados foram analisados pelo programa estatístico Statistical... (2000).

\section{RESULTADOS E DISCUSSÃO}

Os valores de $\mathrm{pH}$ urinário obtidos foram de $6,7 \pm 0,8$ e 5,5 $\pm 0,2$, nos dias 21 e 10 pré-parto, respectivamente, e nenhum animal apresentou duas mensurações consecutivas com valores de $\mathrm{pH}$ superiores a 7,0. Em relação à cetonemia, os valores médios de beta-hidroxibutirato foram de $1,97 \pm 0,84$, aos 21 dias pré-parto, e de $1,81 \pm 0,80$, aos 10 dias pré-parto. Uma vaca, do grupo controle, foi retirada do experimento por apresentar valor de BHBA superior a $3 \mathrm{mmol} / \mathrm{L}$ aos 10 dias antes do parto, considerada com cetose clínica de acordo com a definição de Oetzel (2004).

No período pós-parto, os valores de BHBA foram inferiores aos encontrados no pré-parto, não havendo diferença entre os grupos tratado e controle (Tab. 1). Quanto ao efeito da colina, Piepenbrink e Overton (2003) também não detectaram diferença nos teores de BHBA em animais no periparto em nenhuma das doses testadas. A colina diminui a deposição de gordura no fígado (Zom et al., 2011), maximizando a função hepática e, por consequência, reduzindo a formação de corpos cetônicos e o risco de cetose. Os teores de BHBA foram elevados no presente estudo em comparação aos valores encontrados para animais nos primeiros 30 dias de lactação (Alvarenga et al., 2015). Já os teores de AGNE apresentaram-se reduzidos durante o período experimental. 


\section{Aires et al.}

Tabela 1. Médias ajustadas dos parâmetros sanguíneos e erro-padrão da média de vacas no pós-parto e análise de variância de efeitos fixos tratamento, tempo e interação tempo $x$ tratamento

\begin{tabular}{|c|c|c|c|c|c|c|}
\hline & \multicolumn{3}{|c|}{ Momentos experimentais } & \multicolumn{3}{|c|}{ Valor de $\mathrm{p}^{4}$} \\
\hline Parâmetros ${ }^{1}$ & Dia $10 \mathrm{PP}^{3}$ & Dia $20 \mathrm{PP}^{3}$ & Dia $30 \mathrm{PP}^{3}$ & Tratamento & Tempo & $\begin{array}{c}\text { Tratamento } \\
x \\
\text { Tempo }\end{array}$ \\
\hline $\begin{array}{l}\text { Colesterol } \\
(\mathrm{mg} / \mathrm{dL})\end{array}$ & $\begin{array}{l}63,20 \pm 5,60^{\mathrm{Aa}} \\
77,43 \pm 4,68^{\mathrm{Aa}}\end{array}$ & $\begin{array}{l}62,00 \pm 9,90^{\mathrm{Aa}} \\
64,75 \pm 8,18^{\mathrm{Aa}}\end{array}$ & $\begin{array}{l}95,50 \pm 12,58^{\mathrm{Aa}} \\
113,20 \pm 18,02^{\mathrm{Aa}}\end{array}$ & 0,46 & 0,07 & 0,59 \\
\hline $\begin{array}{l}\text { Frutosamina } \\
(\mu \mathrm{mol} / \mathrm{L})\end{array}$ & $\begin{array}{l}132,55 \pm 11,74^{\mathrm{Aa}} \\
138,82 \pm 13,76^{\mathrm{Aa}}\end{array}$ & $\begin{array}{l}102,36 \pm 11,20^{\mathrm{Aa}} \\
95,82 \pm 12,89^{\mathrm{Aa}}\end{array}$ & $\begin{array}{l}122,76 \pm 10,81^{\mathrm{Aa}} \\
109,70 \pm 6,13^{\mathrm{Aa}}\end{array}$ & 0,59 & 0,24 & 0,54 \\
\hline $\begin{array}{c}\text { BHBA } \\
(\mathrm{mmol} / \mathrm{L})\end{array}$ & $\begin{array}{l}1,12 \pm 0,21^{\mathrm{Aa}} \\
0,90 \pm 0,08^{\mathrm{Aa}}\end{array}$ & $\begin{array}{l}1,55 \pm 0,21^{\mathrm{Aa}} \\
1,46 \pm 0,27^{\mathrm{Aa}}\end{array}$ & $\begin{array}{l}1,17 \pm 0,10^{\mathrm{Aa}} \\
1,59 \pm 0,28^{\mathrm{Aa}}\end{array}$ & 0,21 & 0,15 & 0,2 \\
\hline $\begin{array}{l}\text { AGNE } \\
(\mathrm{mEq} / \mathrm{L})\end{array}$ & $\begin{array}{l}0,24 \pm 0,03^{\mathrm{Aa}} \\
0,32 \pm 0,05^{\mathrm{Aa}}\end{array}$ & $\begin{array}{l}0,19 \pm 0,04^{\mathrm{Aa}} \\
0,15 \pm 0,04^{\mathrm{Ab}}\end{array}$ & $\begin{array}{l}0,21 \pm 0,07^{\mathrm{Aa}} \\
016 \pm 0,02^{\mathrm{Ab}}\end{array}$ & 0,58 & 0,049 & 0,66 \\
\hline $\operatorname{AST}(\mathrm{U} / \mathrm{L})$ & $\begin{array}{l}79,80 \pm 5,30^{\mathrm{Aa}} \\
89,43 \pm 5,08^{\mathrm{Aa}}\end{array}$ & $\begin{array}{l}84,00 \pm 4,75^{\mathrm{Aa}} \\
62,67 \pm 7,30^{\mathrm{Ab}}\end{array}$ & $\begin{array}{l}73,50 \pm 5,17^{\mathrm{Aa}} \\
78,40 \pm 9,64^{\mathrm{Aab}}\end{array}$ & 0,86 & 0,04 & 0,40 \\
\hline GGT (U/L) & $\begin{array}{l}22,20 \pm 1,84^{\mathrm{Aa}} \\
24,57 \pm 1,51^{\mathrm{Aa}}\end{array}$ & $\begin{array}{l}22,50 \pm 3,49^{\mathrm{Aa}} \\
18,50 \pm 2,60^{\mathrm{Aa}}\end{array}$ & $\begin{array}{l}12,25 \pm 2,12^{\mathrm{Aa}} \\
15,40 \pm 1,01^{\mathrm{Aa}}\end{array}$ & 0,69 & 0,20 & 0,82 \\
\hline $\begin{array}{l}\text { IGF-1 } \\
(\mathrm{ng} / \mathrm{mL})\end{array}$ & $\begin{array}{l}41,98 \pm 10,35^{\mathrm{Aa}} \\
60,04 \pm 10,50^{\mathrm{Aa}}\end{array}$ & $\begin{array}{l}45,34 \pm 11,51^{\mathrm{Aa}} \\
58,27 \pm 9,41^{\mathrm{Aa}}\end{array}$ & $\begin{array}{l}113,00 \pm 26,82^{\mathrm{Aa}} \\
75,60 \pm 13,12^{\mathrm{Aa}}\end{array}$ & 0,29 & 0,15 & 0,87 \\
\hline $\begin{array}{c}\text { TOS (mmol } \\
\text { Trolox } \\
\text { Equivalent/L) }\end{array}$ & $\begin{array}{l}58,04 \pm 3,93^{\mathrm{Aa}} \\
64,09 \pm 8,77^{\mathrm{Aa}}\end{array}$ & $\begin{array}{l}60,65 \pm 4,17^{\mathrm{Aa}} \\
53,00 \pm 6,37^{\mathrm{Aa}}\end{array}$ & $\begin{array}{l}68,15 \pm 5,05^{\mathrm{Aa}} \\
66,36 \pm 2,84^{\mathrm{Aa}}\end{array}$ & 0,67 & 0,41 & 0,66 \\
\hline
\end{tabular}

${ }^{1}$ Variáveis: $\mathrm{BHB}$ = beta-hidroxibutirato; AGNE = ácido graxo não esterificado; AST = aspartato aminotransferase; GGT = gamaglutamiltransferase; IGF = fator de crescimento semelhante à insulina; TOS = status oxidante total.

${ }^{2}$ Grupos experimentais: $\mathrm{C}=$ controle; $\mathrm{T}=$ tratado ( $80 \mathrm{~g}$ de colina protegida).

${ }^{3}$ Momentos experimentais: coletas realizadas após 10,20 e 30 dias da data do parto.

${ }^{4}$ Nível de significância para o teste de variância dos efeitos fixos tempo, tratamento e tempo $x$ tratamento.

Dados expressos em média \pm erro-padrão da média; letras maiúsculas diferentes representam significância estatística $(\mathrm{P}<0,05)$ na coluna; letras minúsculas diferentes representam significância estatística $(\mathrm{P}<0,05)$ nas linhas.

Os ácidos graxos não esterificados (AGNE), precursores do BHBA, são derivados das reservas corporais de energia, e a mensuração de seus teores séricos tem sido utilizada para determinar a grau de balanço energético negativo (BEN) em que o animal se encontra (Ospina et al., 2010). Neste estudo, as concentrações desse metabólito foram semelhantes entre os grupos, em todos os tempos experimentais. Entretanto, a colina foi capaz de reduzir os valores dos AGNE nos tempos 20 e 30 do pós-parto em relação aos teores encontrados no dia 10 dos mesmos períodos (Tab. 1). Nenhum animal apresentou teores de AGNE superiores a $0,7 \mathrm{mEq} / \mathrm{L}$, valor associado ao maior risco para ocorrência de doenças no pós-parto de vacas leiteiras (Ospina et al., 2010). Os estudos sobre a suplementação de colina apresentam resultados discrepantes. $\mathrm{Xu}$ et al. (2006) não observaram diferença nos teores de AGNE, mesmo ao suplementarem diferentes doses de colina. No entanto, Pinotti et al. (2003) 
e Cooke et al. (2007) relataram redução significativa nos teores de AGNE no pós-parto de vacas suplementadas com colina, assim como observado ao longo do tempo, no presente estudo, em animais tratados com colina protegida.

Não houve diferença entre os grupos nos teores de frutosamina e de colesterol (Tab. 1). De acordo com Mostafavi et al. (2014), os teores desses dois metabólitos têm relação direta com o metabolismo hepático das gorduras, de tal forma que vacas com teores de frutosamina inferiores a $213 \mu \mathrm{mol} / \mathrm{L}$ têm 4,5 vezes mais chance de apresentar lipidose hepática do que animais com concentrações mais elevadas. Destaca-se que, neste estudo, os valores desse metabólito no pósparto foram menores que $213 \mu \mathrm{mol} / \mathrm{L}$, em todos os animais, assim como os teores de BHBA apresentaram-se elevados, o que poderia indicar uma predisposição a transtornos do metabolismo lipídico nesse rebanho.

Não há estudos sobre a ação da colina nos teores de frutosamina em vacas leiteiras, no entanto sabe-se que essa glicoproteína está diretamente relacionada com os teores de glicose sanguíneos (Ropstad, 1987). De acordo com essa relação, os resultados do presente estudo foram similares ao observados por Zom et al. (2011), que também não detectaram efeito da suplementação com colina nos teores de glicose em vacas leiteiras.

Guretzky et al. (2006) descreveram uma correlação positiva entre os teores de colesterol sérico e o consumo de matéria seca, sendo este um marcador metabólico confiável de ingestão para ruminantes. Não houve influência nas concentrações de colesterol do tratamento com colina protegida (Tab. 1). Em estudo realizado por $\mathrm{Xu}$ et al. (2006), os pesquisadores não relataram mudanças nos teores de colesterol em vacas tratadas com colina protegida. Esse comportamento pode estar relacionado ao consumo de matéria seca, que parece não ser influenciado pela suplementação de colina protegida (Xu et al., 2006; Lima et al., 2012). Os teores de colesterol tenderam a variar de acordo com o tempo $(\mathrm{P}=0,07)$, com teores apresentando decréscimo no $20^{\circ}$ dia e aumento aos 30 dias pós-parto; comportamento semelhante foi descrito por Stengärde et al. (2008).

As enzimas aspartato aminotransferase (AST) e gamaglutamiltransferase (GGT) são utilizadas como importantes marcadores da atividade hepática. Vacas no período de transição, quando sob intenso BEN, apresentam teores elevados dessas enzimas, alteração associada a grande mobilização de gordura corporal e sobrecarga hepática por triglicerídeos (González et al., 2011). No presente estudo, de acordo como os teores de AGNE encontrados, o BEN foi moderado, havendo, portanto, menor mobilização de gordura. Os teores de AST mantiveram-se abaixo daqueles descritos para vacas em transição (Alvarenga et al., 2015), indicando que o BEN moderado não proporcionou alteração hepática expressiva. Não houve diferença nos valores de AST e GGT entre os grupos experimentais (Tab. 1), assim como descrito por Jorritsma et al. (2003). No entanto, em relação ao tempo experimental, houve diferença no grupo tratado, entre os dias 10 e 20 do pós-parto $(\mathrm{P}=0,04)$.

$\mathrm{O}$ fator de crescimento semelhante à insulina 1 (IGF-1) é produzido pelo fígado, por meio da ação da somatotropina, possuindo relação direta com o metabolismo energético hepático e com a atividade reprodutiva (Jorritsma et al., 2003) em vacas. De acordo com Kobayashi et al. (1999), os teores de IGF-1 encontram-se reduzidos, em vacas leiteiras, desde o dia do parto até cerca de 20 dias pós-parto, quando este passa a ser produzido em maior quantidade pelo fígado. Essa mesma tendência foi observada neste estudo com os maiores teores de IGF-1 aos 30 dias pósparto. A suplementação com colina não alterou as concentrações séricas de IGF-1 (Tab. 1). Segundo Betschart et al. (1986), o fornecimento de dieta deficiente em colina reduz o número de receptores de insulina nos hepatócitos, o que, por sua vez, reduz a síntese de IGF-1 pelo fígado. Os teores de IGF-1 mantiveram-se dentro dos limites de referência, mesmo nos animais do grupo controle, o que indica síntese adequada desse hormônio e, consequentemente, presença de teores mínimos de colina para sua produção, não havendo, portanto, efeito ao se suplementar esse nutriente.

De acordo com Schönfeld e Wojtczak (2008), as concentrações de AGNE possuem estreita relação com a quantidade de espécies reativas ao oxigênio (EROs) produzida pelo organismo de vacas leiteiras, de tal forma que vacas sob grave BEN apresentam altos teores desses metabólitos. Os EROs são responsáveis por inúmeras 
alterações na imunidade e na saúde da vaca leiteira (Sordillo et al., 2009). Não houve diferença no status oxidativo entre grupos nem entre os momentos de coleta (Tab. 1). Como os teores de AGNE mantiveram-se baixos, pode-se dizer que não houve desafio suficiente para alterar o perfil oxidativo dos animais.

Não houve influência do tratamento na produção de leite. A média de produção para os dois grupos ao final dos primeiros 30 dias de lactação foi de $26,7 \pm 1,1 \mathrm{~L} /$ dia para o grupo controle e de $25,7 \pm 0,6 \mathrm{~L} /$ dia para o grupo tratado. Resultados semelhantes foram descritos por Davidson et al. (2008) e por Zom et al. (2011). No entanto, Zahra et al. (2006) detectaram um aumento médio de $1,2 \mathrm{~kg}$ nos primeiros 60 dias pós-parto, quando os animais foram suplementados com colina, e Pinotti et al. (2003) descreveram um aumento médio de $2,9 \mathrm{~kg}$ no primeiro mês de lactação em vacas tratadas com colina. Tanto no estudo de Pinotti et al. (2003) quanto no estudo de Zahra et al. (2006), os teores de AGNE do grupo controle estavam acima de $0,5 \mathrm{mEq} / \mathrm{L}$; entretanto, os valores de AGNE obtidos neste estudo e nos experimentos realizados por Davidson et al. (2008) e Zom et al. (2011) foram inferiores a $0,5 \mathrm{mE} / \mathrm{L}$, o que pode indicar que a colina protegida apresenta-se mais eficaz em indivídiuos que experimentam um BEN mais grave, melhorando inclusive o desempenho produtivo desses animais, como ocorreu nos estudos citados.

Tabela 2. Ocorrência de endometrite e intervalo entre parto e concepção (média \pm erro- padrão) de vacas leiteiras suplementadas com colina protegida

\begin{tabular}{ccc}
$\begin{array}{c}\text { Grupos } \\
\text { experimentais }\end{array}$ & $\begin{array}{c}\text { Endometrite aos 60 dias (número de } \\
\text { casos/número de animais) }\end{array}$ & Intervalo parto-concepção (dias) $^{1}$ \\
\hline Controle & $4 / 7$ & $120 \pm 21^{\text {a }}$ \\
Tratado & $1 / 8$ & $140 \pm 34^{\text {a }}$ \\
\hline
\end{tabular}

${ }^{\mathrm{T}}$ Número de dias entre o parto e a data da inseminação com prenhes confirmada e erro-padrão.

Letras diferentes na coluna denotam diferença estatística $(\mathrm{P}<0,05)$.

Quanto à saúde uterina, as vacas do grupo tratado apresentaram menor número de casos de endometrite aos 60 dias pós-parto em comparação aos animas do grupo controle (Tab. 2). Apesar da menor prevalência de problemas reprodutivos em animais suplementados, não houve diferença quanto ao intervalo entre o parto e a concepção entre os grupos. Resultados semelhantes foram obtidos por Lima et al. (2012). A endometrite é responsável por considerável aumento no custo de produção, levando a gastos com tratamento medicamentoso e à redução da eficiência reprodutiva do rebanho. A relação do tratamento com colina e o menor número de casos de endometrite aos 60 dias de lactação não está clara no presente estudo. No entanto, sugere-se que essa relação esteja associada ao efeito da colina sobre a resposta imune. Estudos realizados em outras espécies têm evidenciado que a suplementação de colina aumenta a resposta imune humoral e modula a resposta inflamatória inespecífica (Swain eJohri, 2000; Mehta et al., 2010).
Aos 60 dias pós-parto foram mensurados os teores de IGF-I, colesterol e progesterona, não havendo diferença estatística entre os grupos para os dois primeiros parâmetros (Fig. 1). Alterações no status energético, como BEN intenso, elevados teores de AGNE e BHBA, retardam o retorno da atividade ovariana no pósparto de vacas leiteiras (Jorritsma et al., 2003). Quanto à progesterona, os animais do grupo controle apresentaram maior concentração sérica desse hormônio em comparação ao grupo suplementado $\quad(\mathrm{P}=0,04)$. Levando-se em consideração o resultado dos exames ginecológicos, com maior prevalência de endometrite no grupo controle aos 60 dias, maiores valores de progesterona obtidos neste grupo podem estar relacionados com a presença de corpo lúteo persistente (Bulman e Wood, 1980). Portanto, pode-se dizer que a suplementação com colina reduz a ocorrência de corpo lúteo persistente, reduzindo os teores de progesterona aos 60 dias pós-parto. 


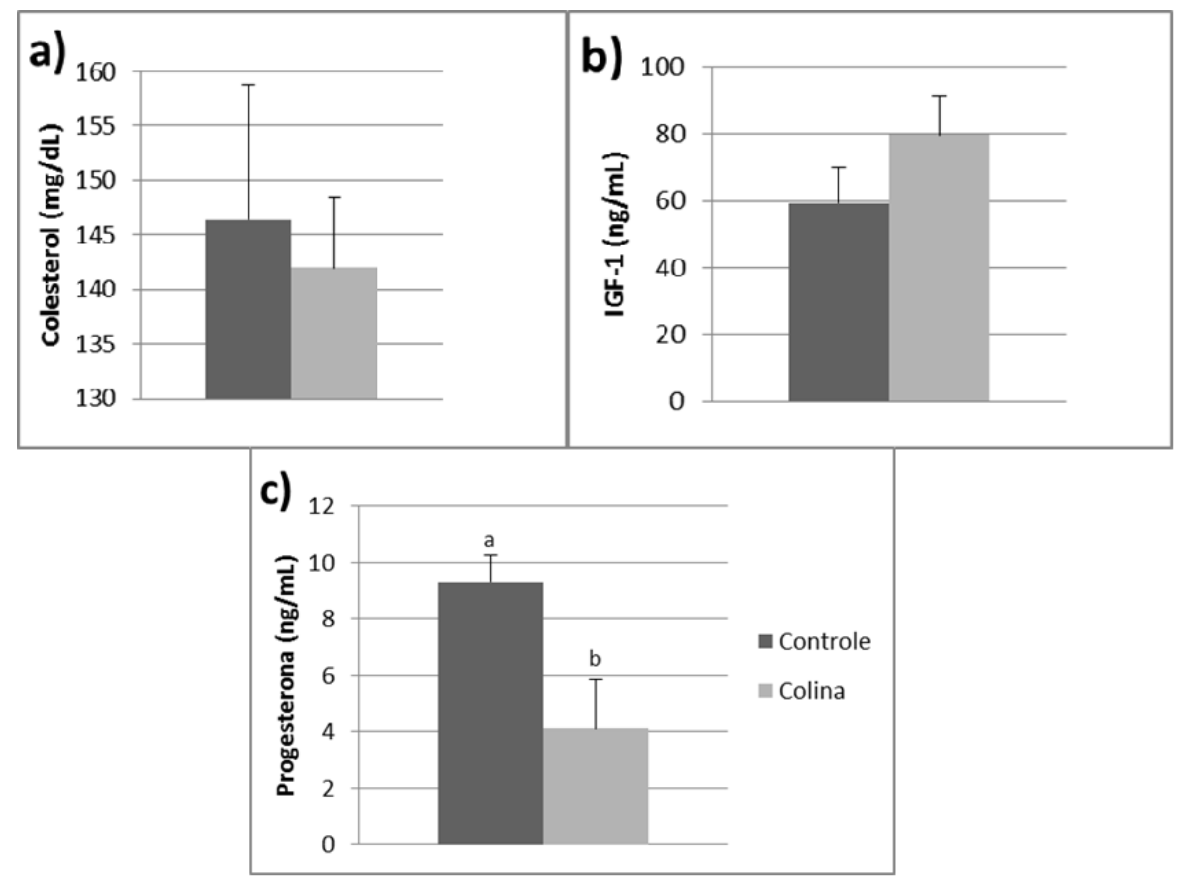

Figura 1. Efeito da suplementação com colina protegida sobre os teores de colesterol, fator de crescimento semelhante à insulina 1 (IGF-1) e progesterona aos 60 dias pós-parto. A) Teores de colesterol sérico aos 60 dias de lactação em animais suplementados ou não com colina protegida. B) Teores de fator de crescimento semelhante à insulina tipo 1 (IGF-1) aos 60 dias de lactação em animais suplementados ou não com colina protegida. c) Teores de progesterona sérico aos 60 dias de lactação em animais suplementados ou não com colina protegida.

Letras diferentes sobre as barras indicam diferença estatística $(\mathrm{P}<0,05)$.

\section{CONCLUSÃO}

A suplementação de colina protegida no pré e no pós-parto não altera os parâmetros bioquímicos de vacas leiteiras nos primeiros 30 dias de lactação, reduzindo, mesmo assim, o número de casos de endometrite aos 60 dias.

\section{REFERÊNCIAS}

ALVARENGA, E.A.; MOREIRA, G.H.F.A.; FACURY FILHO, E.J. et al. Avaliação do perfil metabólico de vacas da raça holandesa durante o período de transição. Pesqui. Vet. Bras., v.35, p.281290, 2015.

BETSCHART, J.M.; VIRJI, M.A.; PERERA, M.I.R.; SHINOZUKA, H. Alterations in hepatocyte insulin receptors in rats fed a choline-deficient diet. Cancer Res., v.46, p.4425-4430, 1986.

BULMAN, D.C.; WOOD, P.D.P. Abnormal patterns of ovarian activity in dairy cows and their relationships with reproductive performance. Anim. Prod., v.30, p.177-188, 1980.
COOKE, R.F.; DEL RIO, N.S.; CARAVIELLO, D.Z. et al. Supplemental choline for prevention and alleviation of fatty liver in dairy cattle. J. Dairy Sci., v.90, p.2413-2418, 2007.

DAVIDSON, S.; HOPKINS, B.A.; ODLE, J. et al. Supplementing limited methionine diets with rumenprotected methionine, betaine, and choline in early lactation Holstein cows. J. Dairy Sci., v.91, p.15521559,2008 .

DRACKLEY, J.K.; OVERTON, T.R.; DOUGLAS, G.N. Adaptations of glucose and long chain fatty acid metabolism in liver of dairy cows during the periparturient period. J. Dairy Sci., v.84, Supl.E, p.100-112, 2001.

DUFFIELD, T.F.; LISSEMORE, K.D.; MCBRIDE, B.W.; LESLIE, K.E. Impact of hyperketonemia in early lactation dairy cows on health and production. $J$. Dairy Sci., v.92, p.571-580, 2009.

EREL, O. A novel automated method to measure total antioxidant response against potent free radical reactions. Clin. Biochem., v.37, p.112-119, 2004. 
GONZÁLEZ, F.D.; MUIÑO, R.; PEREIRA, V. et al. Relationship among blood indicators of lipomobilization and hepatic function during early lactation in high-yielding dairy cows. J. Vet. Sci., v.12, p.251-255, 2011.

GRUNERT, E.; GREGORY, R. (Eds.). Obstetrícia veterinária. 2.ed. Porto alegre: Sulina, 1989. 323p.

GURETZKY, N.A.J.; CARLSON, D.B.; GARRETT, J.E.; DRACKLEY, J.K. Lipid metabolite profiles and milk production for Holstein and Jersey cows fed rumen-protected choline during the periparturient period. J. Dairy Sci., v.89, p.188-200, 2006.

JORRITSMA, R.; WENSING, T.; KRUIP, T.A.M. et al. Metabolic changes in early lactation and impaired reproductive performance in dairy cows. Vet. Res., v.34, p.11-26, 2003.

KOBAYASHI, Y.; BOYD, C.K.; BRACKEN, C.J. et al. Reduced growth hormone receptor (GHR) messenger ribonucleic acid in liver of periparturient cattle is caused by a specific down-regulation of GHR $1^{a}$ that is associated with decreased insulin-like growth factor-I. Endocrinology, v.140, p.3947-3954, 1999.

LIMA, F.S.; SÁ FILHO, M.F.; GRECO, L.F.; SANTOS, J.E.P. Effects of feeding rumen-protected choline on incidence of diseases and reproduction of dairy cows. Vet. J., v.193, p.140-145, 2012.

MEHTA, A.K.; SINGH, B.P.; ARORA, N.; GAUR, S.N. Choline attenuates imune inflammation and suppresses oxidative stress in patients with asthma. Immunobiology, v.215, p.527-534, 2010.

MOSTAFAVI, M.; SEIFI, H.A.; MOHRI, M.; JAMSHIDI, A. Evaluation of fructosamine as a new biomarker for diagnosis of hepatic lipidosis in dairy cows. Anim. Prod. Sci., v.55, p.1005-1010, 2014.

NATIONAL RESEARCH COUNCIL. Nutrient requirements of dairy cattle. 7.ed. Washington: National Academy Press, 2001. 381p.

OETZEL, G.R. Monitoring and testing dairy herds for metabolic diseases. Vet. Clin. Food Anim. Pract., v.20, p.651-674, 2004

OSPINA, P.A.; NYDAM, D.V.; STOKOL, T. et al. Evaluation of nonesterified fatty acids and betahydroxybutyrate in transition dairy cattle in the northeastern United States: critical thresholds for prediction of clinical diseases. J. Dairy Sci., v.93, p.546-554, 2010.
PIEPENBRINK, M.S.; OVERTON, T.R. Liver metabolism and production of cows fed increasing amounts of rumen-protected choline during the periparturient period. J. Dairy Sci., v.86, p.1722-1733, 2003.

PINOTTI, L.; BALDI, A.; POLITIS, I. et al. Rumenprotected choline administration to transition cows: effects on milk production and vitamin E status. J. Vet. Med., v.50, p.18-21, 2003.

ROPSTAD, E. Serum fructosamine levels in dairy cows related to metabolic status in early lactation. Acta Vet. Scand., v.28, p.291-298, 1987.

SCHÖNFELD, P.; WOJTCZAK, L. Fatty acids as modulators of the cellular production of reactive oxygen species. Free Radic. Biol. Med., v.45, p.231241,2008

SORDILLO, L.M.; CONTRERAS, G.A.; AITKEN, S.L. Metabolic factors affecting the inflammatory response of periparturient dairy cows. Anim. Health Res. Rev. v.10, p.53-63, 2009.

STENGÄRDE, L.; TRÅVÉN, M.; EMANUELSON, $\mathrm{U}$. et al. Metabolic profiles in five high-producing Swedish dairy herds with a history of abomasal displacement and ketosis. Acta Vet. Scand., v.50, p.111, 2008.

SWAIN, B.K.; JOHRI, T.S. Effect of supplemental methionine, choline and their combinations on the performance and immune response of broilers. $\mathrm{Br}$. Poult. Sci., v.41, p.83-88, 2000.

XU, G.; YE, J.; LIU, J.; YU, Y. Effect of rumenprotected choline addition on milk performance and blood metabolic parameters in transition dairy cows. Asian Aust. J. Anim. Sci., v.19, p.390-395, 2006.

ZAHRA, L.C.; DUFFIELD, T.F.; LESLIE, K.E. et al. Effects of rumen-protected choline and monensin on milk production and metabolism of periparturient dairy cows. J. Dairy Sci., v.89, p.4808-4818, 2006.

ZEISEL, S.H.; HOLMES-McNARY, M. Choline. In: RUCKER, R.B.; SUTTIE, J.W.; MCCORMICK, D.B. (Eds.). Handbook of vitamins. 3.ed. New York: Marcel Dekker, 2001. p.513-528.

ZOM, R.L.G.; VAN BAAL, J.; GOSELINK , R.M.A. Effect of rumen-protected choline on performance, blood metabolites, and hepatic triacylglycerols of periparturient dairy cattle. J. Dairy Sci., v.94, p.40164027, 2011. 